\title{
Fast Updating of Maximum Likelihood Direction of Arrival Estimates
}

\author{
A. LeE SWindehurst \\ Dept. of Electrical \& Computer Engineering \\ Brigham Young University \\ Provo, Utah 84602
}

\begin{abstract}
The recently introduced weighted subspace fitting (WSF) and MODE algorithms have been shown to provide direction of arrival (DOA) estimates whose variance is asymptotically equivalent to that achievable by the stochastic (unconditional) maximum likelihood method. Updating the WSF and MODE DOA estimates when new data arrives requires recomputation of an eigendecomposition ( $E V D$ ), a numerical operation that is quite complex and does not easily lend itself to parallelization. To overcome this problem, a DOA update algorithm is presented in this paper which has asymptotic properties identical to WSF and MODE, but which requires no EVD. The algorithm achieves computationally efficient estimate updates, and unlike the EVD may be easily parallelized. In addition to describing the algorithm and its asymptotic properties, the paper also includes a simulation example to validate the algorithm's performance.
\end{abstract}

\section{Introduction}

M AXIMUM LIKELIHOOD methods are a standard approach to solving parameter estimation problems such as those encountered in narrowband direction of arrival (DOA) estimation. In the maximum likelihood (ML) approach, the probability distribution of the observations is expressed as a function of the parameters to be estimated, and the so-called (log)likelihood is maximized with respect to these parameters. Under certain regularity conditions, estimates obtained from the ML approach are both asymptotically unbiased and efficient, meaning that they achieve the Cramér-Rao lower bound (CRB) on estimate variance.

Despite its advantages, ML-based methods have not enjoyed much practical application in DOA estimation since they usually lead to non-linear, multidimensional optimization problems that are not easily solved. Even under the "simplest" assumptions of Gaussian signals and noise, maximizing the loglikelihood is quite complicated and does not easily Inc.

${ }^{1}$ This work was supported by the IR\&D Program at ESL, $1058-6393 / 91 \$ 01.00 @ 1991$ IEEE lend itself to numerical solution $[1,2]$. As a result, for many years research has focused on more easily implemented one-dimensional techniques such as beamforming, MUSIC [3], and the min-norm algorithm [4]. While these methods achieve ML or near-ML performance in many cases, difficulties arise when the signals have nearly co-incident DOAs or are highly correlated. These difficulties are a direct result of using a onedimensional optimization procedure to solve what is inherently a multidimensional problem.

Recently, two new multidimensional algorithms have been proposed whose asymptotic second order performance is equivalent to that of ML; i.e., they produce DOA estimates that asymptotically achieve the CRB for Gaussian signals and noise. These algorithms are the weighted subspace fitting (WSF) method of Viberg and Ottersten $[5,6]$, and the method of direction of arrival estimation (MODE) algorithm of Stoica and Sharman $[7,8]$. One of the principal advantages of these techniques over ML is that the form of their respective criterion functions is more amenable to numerical extremization than is the corresponding loglikelihood.

Both WSF and MODE do, however, require that one compute the $d$ principal eigenvectors and eigenvalues of the data covariance matrix, where $d$ is the number of signals present. Initially, this is not a significant issue since all of the eigenvalues of the covariance are needed in determining the value of $d$. Once the number of signals is known, however, and an initial DOA estimate is obtained, updating the WSF and MODE estimates in response to additional data from the array requires that the principal eigenspace be completely recomputed. Algorithms have been proposed for performing the eigenspace update efficiently $[9,10,11,12,13]$, but such techniques are still quite complex and often suffer from a linear buildup of round-off error.

The goal of this paper is to demonstrate that asymptotically efficient DOA estimates can be obtained without resorting to an eigendecomposition. In particular, an algorithm similar in form to WSF and possessing identical asymptotic properties will be de- 
veloped that requires computation of only the sample covariance and its inverse. While computing the inverse of an $m \times m$ matrix takes $O\left(\mathrm{~m}^{3}\right)$ operations in general and hence would not seem to provide much of a computational advantage, updating the inverse of a matrix after a rank-one modification is a trivial $O\left(\mathrm{~m}^{2}\right)$ calculation. Hence, the new algorithm is well-suited to the problem of updating the DOA estimates when a new sample, or snapshot, is taken from the array.

After introducing notation and describing the WSF approach in the next section, the new algorithm is presented in Section 3 and its asymptotic equivalence to WSF is established. Some important features of the algorithm are outlined in Section 4, and a simulation example is included in Section 5.

\section{Weighted Subspace Fitting}

Under the assumption of narrowband signals, the output of an $m$-element array $\mathbf{x}(t) \in \mathbb{C}^{m}$ due to $d$ sources can be described by

$$
\mathbf{x}(t)=\mathbf{A}\left(\boldsymbol{\theta}_{0}\right) \mathbf{s}(t)+\mathbf{n}(t),
$$

where $\mathbf{s}(t) \in \mathbb{C}^{d}$ represents the signal amplitude and phase at time $t, \mathbf{n}(t) \in \mathbb{C}^{m}$ is additive noise, and $\mathbf{A}\left(\boldsymbol{\theta}_{0}\right) \in \mathbb{C}^{\boldsymbol{m \times d}}$ is the matrix of array response vectors for each source parameterized by the DOAs $\theta_{0}=$ $\left[\theta_{1}, \cdots, \theta_{d}\right]^{T}$ :

$$
\mathbf{A}\left(\boldsymbol{\theta}_{0}\right)=\left[\begin{array}{lll}
\mathbf{a}\left(\theta_{1}\right) & \cdots & \mathbf{a}\left(\theta_{d}\right)
\end{array}\right] .
$$

In this analysis, it is assumed that the array parameterization $\mathbf{a}(\theta)$ is completely known (i.e., the array is fully calibrated), and is unambiguous (i.e., every matrix of the form (2) is full rank $d$ for distinct $\theta_{i}$ ). It will also be assumed without loss of generality that the noise is spatially white, or in other words that $\mathcal{E}\left\{\mathbf{n}(t) \mathbf{n}^{*}(t)\right\}=\sigma^{2} \mathbf{I}$. If the noise is further assumed to be uncorrelated with the signals, then the covariance of the array data is given by

$$
\mathbf{R} \stackrel{\text { def }}{=} \mathcal{E}\left\{\mathbf{x}(t) \mathbf{x}^{*}(t)\right\}=\mathbf{A}\left(\boldsymbol{\theta}_{0}\right) \mathbf{S} \mathbf{A}^{*}\left(\boldsymbol{\theta}_{0}\right)+\sigma^{2} \mathbf{I},
$$

where $\mathbf{S} \stackrel{\text { def }}{=} \mathcal{E}\left\{\mathbf{s}(t) \mathbf{s}^{*}(t)\right\}$ is the covariance of the emitter signals. The goal of the narrowband DOA estimation problem is simply to use several observations from the array and exploit the model of (3) to estimate $\boldsymbol{\theta}$.

To implement an ML solution to this problem, the likelihood function of the data must first be determined and then maximized over all relevant model parameters. If the signal and noise waveforms $\mathbf{s}(t)$ and $\mathbf{n}(t)$ are assumed to be stationary, temporally whitc. zero-mean complex Gaussian random processes, then maximizing the log-likelihood over $\theta$ can be shown to be equivalent $t^{2}[1,2]$

$$
\min _{\theta} \log \left(\operatorname{det}\left[\mathbf{P}_{A} \hat{\mathbf{R}} \mathbf{P}_{A}-\frac{\operatorname{Tr}\left(\mathbf{P}_{A}^{\perp} \hat{\mathbf{R}}\right)}{m-d} \mathbf{P}_{A}^{\perp}\right]\right),
$$

where $\hat{\mathbf{R}}$ is a sample estimate of (3), and the projection operator $\mathbf{P}_{\boldsymbol{A}}=\mathbf{I}-\mathbf{P}_{\boldsymbol{A}}^{\perp}$ is defined as

$$
\mathbf{P}_{\boldsymbol{A}}=\mathbf{A}(\boldsymbol{\theta})\left[\mathbf{A}^{*}(\boldsymbol{\theta}) \mathbf{A}(\boldsymbol{\theta})\right]^{-1} \mathbf{A}^{*}(\boldsymbol{\theta}) .
$$

This minimization in (4) is sometimes referred to as the stochastic or unconditional ML approach. While the estimates obtained from (4) will have very desirable properties, the determinant operation makes implementing this minimization quite complicated.

In the WSF approach of Viberg and Ottersten [5], the DOA estimates are obtained from the following much simpler minimization problem:

$$
\begin{aligned}
\hat{\boldsymbol{\theta}}_{W S_{F}} & =\arg \min _{\boldsymbol{\theta}, T} \hat{V}_{W S_{F}}(\boldsymbol{\theta})=\left\|\hat{\mathbf{E}}_{s} \mathbf{W}^{\frac{1}{2}}-\mathbf{A}(\boldsymbol{\theta}) \mathbf{T}\right\|_{F}^{2} \\
& =\arg \min _{\theta} \operatorname{Tr}\left(\mathbf{P}_{A}^{\perp} \hat{\mathbf{E}}_{s} \mathbf{W} \hat{\mathbf{E}}_{s}^{*}\right),
\end{aligned}
$$

where $\hat{\boldsymbol{\theta}}_{W S F}$ is the estimated angle vector, $\hat{\mathbf{E}}_{\boldsymbol{s}}$ is a set of vectors obtained from the data which approximately span the "signal" subspace $\operatorname{span}\left\{\mathbf{A}\left(\boldsymbol{\theta}_{0}\right) \mathbf{S}\right\}$, and $\mathbf{W}=\mathbf{W}^{*}$ is a positive definite weighting matrix. Ottersten and Viberg showed that for a particular choice of $\mathbf{W}$, the variance of the estimation error $\hat{\boldsymbol{\theta}}_{W S F}-\boldsymbol{\theta}_{0}$ of (5) is minimized and in fact asymptotically (for large $N$ ) achieves the Cramér-Rao lower bound under the assumption of Gaussian signals and noise. WSF is thus asymptotically equivalent to (4), but it is much simpler to work with since it involves a trace operation instead of a determinant. Note that both algorithms are multidimensional in the sense that the elements of the vector $\hat{\theta}$ are searched for "simultaneously." In addition, an estimate of reasonable quality is also required in either case to initialize the search for the DOAs.

The vectors $\hat{\mathbf{E}}_{\text {s }}$ used in (5) can be obtained from an eigendecomposition of the sample covariance $\hat{\mathbf{R}}$ of the array data:

$$
\begin{aligned}
\hat{\mathbf{R}} & =\frac{1}{N} \sum_{k=1}^{N} \mathbf{x}\left(t_{k}\right) \mathbf{x}\left(t_{k}\right)^{*} \\
& =\sum_{i=1}^{m} \hat{\lambda}_{i} \hat{\mathrm{e}}_{i} \hat{\mathrm{e}}_{i}^{*}
\end{aligned}
$$

\footnotetext{
${ }^{2}$ Note that equation (4) is expressed in concentrated form, where the separable parameters $\sigma^{2}$ and the non-redundant elements of $\mathbf{S}$ have been solved for.
} 


$$
\begin{aligned}
& =\hat{\mathbf{E}} \hat{\mathbf{\Lambda}} \hat{\mathbf{E}}^{*} \\
& =\hat{\mathbf{E}}_{s} \hat{\mathbf{\Lambda}}_{s} \hat{\mathbf{E}}_{s}^{*}+\hat{\mathbf{E}}_{n} \hat{\mathbf{\Lambda}}_{n} \hat{\mathbf{E}}_{n}^{*},
\end{aligned}
$$

where $N$ is the number of observations, or "snapshots," taken from the array, and where $\hat{\boldsymbol{\Lambda}}=$ $\operatorname{diag}\left\{\hat{\lambda}_{1} \geq \hat{\lambda}_{2} \geq \cdots \geq \hat{\lambda}_{m}\right\}$ and $\mathbf{E}=\left[\hat{\mathbf{e}}_{1}, \cdots, \hat{\mathbf{e}}_{m}\right]$ are respectively the eigenvalues and eigenvectors of $\hat{\mathbf{R}}$. If there are no perfectly correlated signals, then

$$
\begin{aligned}
& \hat{\mathbf{E}}_{s}=\left[\hat{\mathbf{e}}_{1} \cdots \hat{\mathbf{e}}_{d}\right] \\
& \hat{\mathbf{E}}_{n}=\left[\hat{\mathbf{e}}_{d+1} \cdots \hat{\mathbf{e}}_{m}\right] \\
& \hat{\mathbf{\Lambda}}_{s}=\operatorname{diag}\left\{\hat{\lambda}_{1}, \cdots, \hat{\lambda}_{d}\right\} \\
& \hat{\boldsymbol{\Lambda}}_{n}=\operatorname{diag}\left\{\hat{\lambda}_{d+1}, \cdots, \hat{\lambda}_{m}\right\} .
\end{aligned}
$$

With these definitions, the optimal choice for $\mathbf{W}$ in (5) was shown in [5] to be

$$
\mathbf{W}=\hat{\mathbf{W}}_{o}=\left(\hat{\mathbf{\Lambda}}_{s}-\hat{\boldsymbol{\sigma}}^{2} \mathbf{I}\right)^{2} \hat{\mathbf{\Lambda}}_{s}^{-1},
$$

where $\hat{\sigma}$ is a consistent estimate of the noise power. When perfectly coherent signals are present, i.e., when $\operatorname{rank}(\mathbf{S})=d^{\prime}<d$, then the low rank signal subspace is only of dimension $d^{\prime}$, and $\mathbf{E}_{\text {s }}$ and $\hat{\boldsymbol{\Lambda}}$ will contain only the first $d^{\prime}$ eigenvectors and eigenvalues of $\hat{\mathbf{R}}$. The weighting $\hat{\mathbf{W}}_{o}$ is $d^{\prime} \times d^{\prime}$ in such cases, and provided $d$ and $d^{\prime}$ are known and $m>2 d-d^{\prime}[14,15]$, WSF is still able to obtain unique, minimum variance DOA estimates.

When additional data is obtained from the array, and an updated DOA estimate is desired, a new set of eigenvectors $\hat{\mathbf{E}}_{\text {s }}$ and a new weighting matrix $\hat{\mathbf{W}}_{o}$ must be computed in order to implement the WSF minimization of (5). Since only the $d^{\prime}$-dimensional principal eigenspace is needed, this computation requires $O\left(m^{2} d^{\prime}\right)$ operations in general. However, the computation is iterative and relatively complex, and hence is not well-suited to a direct parallel implementation. Thus, in situations where the emitters are rapidly moving and the DOA estimates must be frequently updated, or simply when it is desired to account for an additional observation from the array, recomputing the WSF solution may be prohibitively costly.

\section{An Efficient Update Algorithm}

The principal objective of this paper is to demonstrate that DOA estimates obtained from the minimization problem

$$
\hat{\boldsymbol{\theta}}=\arg \min _{\boldsymbol{\theta}} \hat{V}(\boldsymbol{\theta})=\arg \min _{\boldsymbol{\theta}} \operatorname{Tr}\left[\mathbf{P}_{\boldsymbol{A}}^{\perp}\left(\hat{\mathbf{R}}+\hat{\sigma}^{4} \hat{\mathbf{R}}^{-1}\right)\right]
$$

have asymptotic properties identical to those obtained from WSF and the stochastic ML algorithm, and hence are asymptotically unbiased and statistically efficient. If

$$
\begin{aligned}
\mathbf{C}_{W S F} & =\mathcal{E}\left\{\left(\hat{\boldsymbol{\theta}}_{W S F}-\boldsymbol{\theta}_{0}\right)\left(\hat{\boldsymbol{\theta}}_{W S F}-\boldsymbol{\theta}_{0}\right)^{\boldsymbol{T}}\right\} \\
\mathbf{C} & =\mathcal{E}\left\{\left(\hat{\boldsymbol{\theta}}-\boldsymbol{\theta}_{0}\right)\left(\hat{\boldsymbol{\theta}}-\boldsymbol{\theta}_{0}\right)^{\boldsymbol{T}}\right\}
\end{aligned}
$$

are the estimation error covariances for (5) and (7), then this amounts to showing that

$$
\mathbf{C}=\mathbf{C}_{W S F}+o\left(N^{-1}\right),
$$

which in turn amounts to showing that [5]

$$
\begin{aligned}
& \left.\hat{V}^{\prime}\left(\boldsymbol{\theta}_{0}\right) \stackrel{\text { def }}{=} \frac{\partial \hat{V}(\boldsymbol{\theta})}{\partial \boldsymbol{\theta}}\right|_{\theta=\theta_{0}}=\hat{V}_{\boldsymbol{W S F}}^{\prime}\left(\boldsymbol{\theta}_{0}\right)+o\left(N^{-1 / 2}\right)(9) \\
& \left.\hat{V}^{\prime \prime}\left(\boldsymbol{\theta}_{0}\right) \stackrel{\text { def }}{=} \frac{\partial \hat{V}(\boldsymbol{\theta})}{\partial \boldsymbol{\theta} \partial \boldsymbol{\theta}^{T}}\right|_{\theta=\theta_{0}}=\hat{V}_{\boldsymbol{W S F}}^{\prime \prime}\left(\boldsymbol{\theta}_{0}\right)+o(1) . \quad(10)
\end{aligned}
$$

These facts are established in the course of proving the following theorem:

Theorem 3.1 If $\hat{\sigma}^{2}$ is a consistent estimator of the noise power $\sigma^{2}$, then the DOA estimates obtained from (7) are consistent and have the same asymptotic variance as those obtained from the WSF minimization of (5).

Proof: To begin with, note that the estimates obtained from (7) are unchanged if the constant $-2 \hat{\sigma}^{2}(m-d)$ is added to $\hat{V}(\boldsymbol{\theta})$ :

$$
\begin{aligned}
\min _{\theta} \hat{V}(\boldsymbol{\theta}) & =\min _{\theta} \operatorname{Tr}\left[\mathbf{P} \frac{1}{A}\left(\hat{\mathbf{R}}+\hat{\sigma}^{4} \hat{\mathbf{R}}^{-1}\right)\right]-2 \hat{\sigma}^{2}(m-d) \\
& =\min _{\theta} \operatorname{Tr}\left[\mathbf{P}_{A}^{\perp}\left(\hat{\mathbf{R}}+\hat{\sigma}^{4} \hat{\mathbf{R}}^{-1}-2 \hat{\sigma}^{2} \mathbf{I}\right)\right] \cdot(11)
\end{aligned}
$$

Since

$$
\hat{\mathbf{R}}^{-1}=\hat{\mathbf{E}}_{s} \hat{\mathbf{\Lambda}}_{s}^{-1} \hat{\mathbf{E}}_{s}^{*}+\hat{\mathbf{E}}_{n} \hat{\mathbf{\Lambda}}_{n}^{-1} \hat{\mathbf{E}}_{n}^{*}
$$

$\hat{V}(\theta)$ can be further rewritten as

$$
\begin{aligned}
\hat{V}(\boldsymbol{\theta}) & =\operatorname{Tr}\left[\mathbf{P}_{A}^{\perp} \hat{\mathbf{E}}\left(\hat{\boldsymbol{\Lambda}}-\hat{\boldsymbol{\sigma}}^{2} \mathbf{I}\right)^{2} \hat{\boldsymbol{\Lambda}}^{-1} \hat{\mathbf{E}}^{*}\right] \\
& =\hat{V}_{W S F}(\boldsymbol{\theta})+\operatorname{Tr}\left[\mathbf{P}_{\boldsymbol{A}}^{\perp} \hat{\mathbf{E}}_{n} \hat{\mathbf{W}}_{n} \hat{\mathbf{E}}_{n}^{*}\right]
\end{aligned}
$$

where $\hat{\mathbf{W}}_{n}=\left(\hat{\boldsymbol{\Lambda}}_{n}-\hat{\sigma}^{2} \mathbf{I}\right)^{2} \hat{\mathbf{\Lambda}}_{n}^{-1}$, and thus the cost function is still guaranteed to be positive. Since $\hat{\mathbf{W}}_{n}=O\left(N^{-1}\right)$ when $\hat{\sigma}^{2}$ is consistent [16], then

$$
\lim _{N \rightarrow \infty} \hat{V}(\boldsymbol{\theta})=\hat{V}_{W S F}(\boldsymbol{\theta}) \text {. }
$$

The consistency of (7) is established by the fact that WSF is consistent, and by the continuity of both 
$\hat{V}(\theta)$ and $\hat{V}_{W S F}(\theta)$ [5]. Along with (13), the fact that $\hat{\mathbf{w}}_{n}=O\left(N^{-1}\right)$ also guarantees that the error terms in equations (9) and (10) are at least $o\left(N^{-1 / 2}\right)$. Equation (8) thus holds, and the variance of the estimates obtained from (7) is thus asymptotically equivalent to that of WSF.

\section{Discussion}

The most salient feature of (7) is that it requires no eigendecomposition. This may at first appear to be a moot point since evaluating $\hat{V}(\theta)$ does require that $\hat{\mathbf{R}}^{-1}$ be computed. However, updating $\hat{\mathbf{R}}^{-1}$ in response to additional data is considerably less complicated than updating the corresponding dominant eigenspace. For example, suppose $\hat{\mathbf{R}}_{N}$ represents the value of the array covariance after $N$ snapshots, and suppose an additional observation $\mathbf{z}=\mathbf{x}(N+1)$ is received from the array. If the following consistent estimator of $\sigma^{2}$ is used ${ }^{3}$

$$
\hat{\sigma}_{N}^{2}=\frac{1}{m-d} \operatorname{Tr}\left(\mathbf{P}_{A}^{\perp} \hat{\mathbf{R}}_{N}\right),
$$

then the data-dependent quantities in (7) may be updated as follows:

$$
\begin{aligned}
\hat{\mathbf{R}}_{N+1} & =\frac{N}{N+1}\left[\hat{\mathbf{R}}_{N}+\frac{1}{N} \mathbf{z z}^{*}\right] \\
\hat{\mathbf{R}}_{N+1}^{-1} & =\frac{N+1}{N}\left[\hat{\mathbf{R}}_{N}^{-1}-\frac{\hat{\mathbf{R}}_{N}^{-1} \mathbf{z z}^{*} \hat{\mathbf{R}}_{N}^{-1}}{N+\mathbf{z}^{*} \hat{\mathbf{R}}_{N}^{-1} \mathbf{z}}\right] \\
\hat{\sigma}_{N+1}^{2} & =\frac{N}{N+1} \hat{\sigma}_{N}^{2}+\frac{\mathbf{z}^{*} \mathbf{P}_{A}^{1} \mathbf{z}}{(m-d)(N+1)} .
\end{aligned}
$$

In situations where the emitters may be rapidly moving and it is desirable to exponentially decrease the contribution of previous snapshots, a constant $0<$ $\mu<1$ may be defined and the data-dependent updates obtained as follows:

$$
\begin{aligned}
\hat{\mathbf{R}}_{N+1} & =\mu \hat{\mathbf{R}}_{N}+(1-\mu) \mathbf{z} \mathbf{z}^{*} \\
\hat{\mathbf{R}}_{N+1}^{-1} & =\mu^{-1} \hat{\mathbf{R}}_{N}^{-1}-\frac{(1-\mu) \hat{\mathbf{R}}_{N}^{-1} \mathbf{z z}^{*} \hat{\mathbf{R}}_{N}^{-1}}{\mu\left[\mu+(1-\mu) \mathbf{z}^{*} \hat{\mathbf{R}}_{N}^{-1} \mathbf{z}\right]} \\
\hat{\sigma}_{N+1}^{2} & =\mu \hat{\sigma}_{N}^{2}+\frac{(1-\mu) \mathbf{z}^{*} \mathbf{P}}{m-\mathbf{z}} .
\end{aligned}
$$

In either case, updating the data-dependent term in the cost function involves a simple, non-iterative $O\left(\mathrm{~m}^{2}\right)$ calculation that can be easily parallelized.

\footnotetext{
${ }^{3}$ Since the implementation of multidimensional minimizations like WSF and (7) requires reasonably good initial estimates, there is no loss of generality in assuming $\mathbf{P}_{A} \frac{1}{A}$ is available in computing $\hat{\sigma}_{N}^{2}$.
}

In the proof of Theorem 3.1 it was shown that $\hat{V}(\theta)$ is essentially equivalent to

$$
\operatorname{Tr}\left(\mathbf{P}_{\boldsymbol{A}}^{\perp} \hat{\mathbf{E}}\left(\hat{\mathbf{\Lambda}}-\hat{\sigma}^{2} \mathbf{I}\right)^{2} \hat{\mathbf{\Lambda}}^{-1} \hat{\mathbf{E}}^{*}\right) .
$$

Comparison of this expression with (5) and (6) shows that (7) can be thought of as implementing a WSF minimization using all of the eigenvectors and not just the first $d^{\prime}$. The asymptotic equivalence of (14) and $\hat{V}_{W S F}(\theta)$ is not surprising since the weights multiplying $\mathbf{e}_{d^{\prime}+1}, \cdots, \mathbf{e}_{m}$ in (14) are near zero for large $N$. Note also that the minimization of (7) requires only that $d$ and not $d^{\prime}$ be known. Although it is much more difficult to determine $d$ than $d^{\prime}$ when $d^{\prime}<d$, not having to continually estimate $d^{\prime}$ at each update is advantageous in situations where emitters may be fading in and out of coherence.

While adding the constant $-2 \hat{\sigma}^{2}(m-d)$ to $\hat{V}(\theta)$ in (11) does not change the resulting DOA estimates, it makes the minimization more amenable to a GaussNewton (GN) search. This is because the constant forces $\hat{V}(\theta) \simeq 0$ in the vicinity of the true solution, which is an assumption that the GN technique relies on (e.g., see $[6,17])$. For each GN iteration, computing the gradient and approximate Hessian of the cost function in (11) requires $O\left(\mathrm{~m}^{2} d\right)$ operations, compared with $O\left(m d^{2}\right)$ operations for WSF. On the other hand, updating the corresponding data-dependent quantities takes $O\left(m^{2}\right)$ for (11) and $O\left(m^{2} d\right)$ for WSF. Thus, strictly speaking, both algorithms require roughly the same level of computational effort in obtaining an updated DOA estimate via GN. The advantage of (11) lies in the simplicity of its implementation; all of its $O\left(m^{2} d\right)$ and $O\left(m^{2}\right)$ computations are simple matrix/vector products, and are easily parallelized.

If in (11) $\hat{\sigma}^{2}$ is treated as a parameter to be estimated rather than as a consistent estimate of $\sigma^{2}$, then a somewhat different consistent estimator of $\sigma^{2}$ is obtained:

$$
\hat{\sigma}^{2}=\frac{m-d}{\operatorname{Tr}\left(\mathbf{P}_{A}^{\perp} \hat{\mathbf{R}}^{-1}\right)} .
$$

If this estimate is substituted back into (11), the following concentrated version of the cost function results:

$$
\hat{V}(\boldsymbol{\theta})=\operatorname{Tr}\left(\mathbf{P}_{A}^{\perp} \hat{\mathbf{R}}\right)-\frac{(m-d)^{2}}{\operatorname{Tr}\left(\mathbf{P}_{A}^{\perp} \hat{\mathbf{R}}^{-1}\right)} .
$$

Equation (15) thus provides another "eigendecomposition-free" alternative to WSF. It is interesting to note the similarity of the cost functions in (7) and (15) with that of the so-called deterministic or conditional ML algorithm (CML), which implements

$$
\hat{\boldsymbol{\theta}}=\arg \min _{\boldsymbol{\theta}} \hat{V}_{C M L}(\boldsymbol{\theta})=\arg \min _{\theta} \operatorname{Tr}\left(\mathbf{P} \frac{1}{A} \hat{\mathbf{R}}\right) .
$$


The CML approach differs from unconditional ML in that it treats samples of the signal waveforms as deterministic parameters instead of random variables. Ottersten and Viberg have shown that WSF asymptotically outperforms CML independent of whether the signals are deterministic or stochastic [18]. The analysis above demonstrates that the estimates obtained from (7) will also have asymptotically lower variance than CML.

\section{A Simulation Example}

This purpose of this example is simply to demonstrate that the algorithms described in (5) and (11) have essentially the same performance, and that they both significantly outperform CML. A 12-element uniform circular array with unity gain sensors and a 1- $\lambda$ radius was assumed in this example, and two emitters were simulated at angles of $0^{\circ}$ and $10^{\circ}$ with respect to a line through one of the sensors. The emitter signals were generated as constant amplitude planewaves with random phase, uniformly distributed on $[0,2 \pi]$. The $0^{\circ}$ source had an SNR of $0 \mathrm{~dB}$, while that of the $10^{\circ}$ source was $20 \mathrm{~dB}$.

In each trial, an estimate based on 500 snapshots was obtained for WSF, CML, and the minimization of (11). A total of 1000 trials were conducted for each of the correlation coefficient values $0.8,0.9,0.95$, and 0.99 , and the standard deviation of the estimates was computed. The results are shown in Figure 1, where the connected lines indicate theoretical predictions of the estimate standard deviation (see [5]), and the symbols '*', ' $x$ ', and 'o' indicate sample estimates computed from the simulations. Note that the acronym MUD stands for Maximum likelihood UpDate, and refers to the minimization of (11).

\section{References}

[1] J. F. Böhme, "Estimation of Spectral Parameters of Correlated Signals in Wavefields", Signal Processing, 10:329 $337,1986$.

[2] A. G. Jaffer, "Maximum Likelihood Direction Finding of Stochastic Sources: A Separable Solution", In Proc. ICASSP '88, pages 2893-2896, New York, N.Y., 1988.

[3] R. Schmidt, A Signal Subspace Approach to Multiple Emitter Location and Spectral Estimation, $\mathrm{PhD}$ thesis, Stanford University, 1981.

[4] R. Kumaresan and D. Tufts, "Estimating the Angle of Arrival of Multiple Plane Waves", IEEE Trans. on AES, 19:134-139, 1983.

[5] M. Viberg and B. Ottersten, "Sensor Array Processing Based on Subspace Fitting", IEEE Trans. on Sig. Proc., 39(5):1110-1121, May 1991.

[6] M. Viberg, B. Ottersten, and T. Kailath, "Detection and Estimation in Sensor Arrays Using Weighted Subspace Fitting", IEEE Trans. on Sig. Proc., 39(11), Nov. 1991.

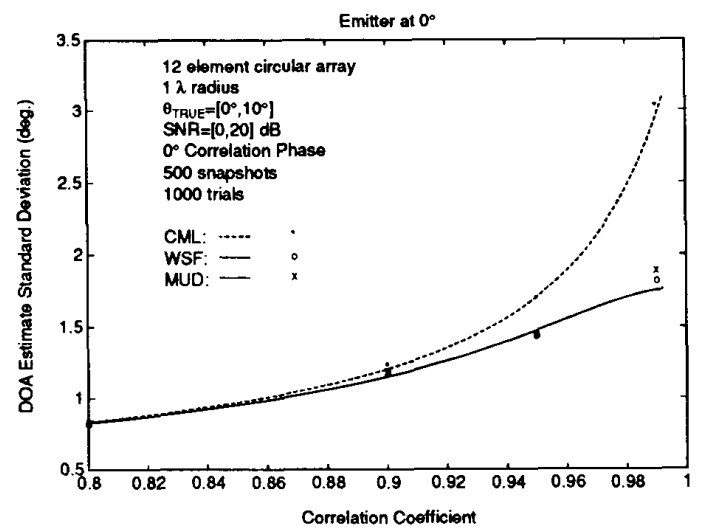

Figure 1: Algorithm Performance vs. Correlation Coefficient

[7] P. Stoica and K. Sharman, "Maximum Likelihood Methods for Direction-of-Arrival Estimation", IEEE Trans. on ASSP, 38(7):1132-1143, July 1990.

[8] P. Stoica and A. Nehorai, "Performance Study of Conditional and Unconditional Direction-of-Arrival Estimation", IEEE Trans. on ASSP, 38(10):1783-1795, October 1990.

[9] J. R. Bunch, C. P. Nielsen, and D. C. Sorensen, "RankOne Modification of the Symmetric Eigenproblem", $\mathrm{Nu}$ merische Mathematik, 31:31-48, 1978.

[10] I. Karasalo, "Estimating the Covariance Matrix by Signal Subspace Averaging", IEEE Trans. on ASSP, 34(1):8-12, January 1986.

[11] R. Schreiber, "Implementation of Adaptive Array Algorithms", IEEE Trans. on ASSP, 34(5):1038-1045, October 1986.

[12] R. D. DeGroat and R. A. Roberts, "Efficient, Numerically Stabilized Rank-One Eigenstructure Updating", IEEE Trans. on $A S S P$, 38(2):301-316, February 1990.

[13] K. Yu, "Recursive Updating the Eigenvalue Decomposition of a Covariance Matrix", IEEE Trans. on ASSP, 39(5):1136-1145, May 1991.

[14] Y. Bresler and A. Macovski, "On the Number of Signals Resolvable by a Uniform Linear Array", IEEE Trans. on ASSP, 34(6):1361-1375, December 1986.

[15] M. Wax and I. Ziskind, "On Unique Localization of Multiple Sources by Passive Sensor Arrays", IEEE Trans. ASSP, ASSP-37(7):996-1000, July 1989.

[16] P. Stoica and A. Nehorai, "MUSIC, Maximum Likelihood, and Cramér-Rao Bound", IEEE Trans. on ASSP, 37(5):720-741, May 1989.

[17] A. Swindlehurst, B. Ottersten, R. Roy, and T. Kailath, "Multiple Invariance ESPRIT", to appear in IEEE Trans. on Sig. Proc., 40, April 1992.

[18] B. Ottersten and M. Viberg, "Asymptotic Robustness of Sensor Array Processing Methods", In Proc. IEEE ICASSP, volume 5, pages 2635-2638, Albuquerque, NM, 1990. 